\title{
Assessment of knowledge, attitude and practices and analysis on risk factor regarding schistosomiasis among fishermen and boatmen in Dongting Lake Basin, P.R. China
}

\section{Zhou Guan}

National Institute of parasitic diseases, Chinese Center for Disease Control and Prevention

\section{Si-Min Dai}

National Institute of Parasitic Diseases, Chinese Center for Disease Control and Prevention Jie Zhou

Hunan Institute of Schistosomiasis Control

\section{Xiao-Bing Ren}

Yueyang County Office for Preventive and Control on Schistosomiasis

\section{Zhi-Qiang Qin}

National Institute of Parasistic Diseases, Chinese Center for Disease Control and Prevention

Yin-Long Li

National Institute of Parasitic Diseases, Chinese Center for Disease Control and Prevention

Shan LV

National Institute of Parasitic Diseases, Chinese Center for Disease Control and Prevention

Shi-Zhu Li

National Institute of Parasistic Diseases, Chinese Center for Disease Control and Prevention

\section{Xiao-Nong Zhou}

National Institute of Parasitic Diseases, Chinese Center for Disease Control and Prevention

Jing Xu ( $\nabla$ xfmjing@163.com )

National Institute of parasitic diseases, Chinese Center for Disease Control and Prevention

\section{Research}

Keywords: Schistosomiasis, Knowledge, Attitude, Practice, Prevalence, Influence factors, Logistic regression

Posted Date: December 6th, 2019

DOI: https://doi.org/10.21203/rs.2.18362/v1 
License: (c) (i) This work is licensed under a Creative Commons Attribution 4.0 International License. Read Full License

Version of Record: A version of this preprint was published at Parasites \& Vectors on June 1st, 2020. See the published version at https://doi.org/10.1186/s13071-020-04157-4. 


\section{Abstract}

Background: Fishermen and boatmen are high risk population to get infection by schistosomes due to their high frequency water contact in endemic areas of schistosomiasis in the People's Republic of China (P. R. China). To develop specific interventions towards this population, understanding their knowledge, attitude and practices (KAPs) towards schistosomiasis and exploring risk factors for schistosomiasis are necessary. Therefore, our present study was designed to assess the KAPs of fishermen and boatmen and to identify the risk factors associated with schistosome infection based on a cross-sectional survey in selected area.

Methods: A cross section survey was conducted in Dongting Lake Basin of Yueyang County, Hunan Province, P. R. China. A total of 601 fishermen and boatmen were interviewed from October to November in 2017. Information regarding socio-demographic details and knowledge, attitude, and practices toward schistosomiasis were collected through a standardized questionnaire. Meanwhile, fecal samples of participants were collected and detected by Polymerase Chain Reaction (PCR) technique to understand the infection status of schistosomes among fishermen and boatmen.

Results: Of the 601 interviewed participants, more than 90\% respondents knew the way of getting infection, intermediate host of schistosomes and preventive methods, while majority of the respondents knew this disease and its cause, clinical manifestations and transmission mode properly. The majority of the respondents had positive attitude towards schistosomiasis prevention. However, only $6.66 \%$ respondents had installed latrine on their boats, while $32.61 \%$ respondents defecated in the public toilets on shore. In addition, only $4.99 \%$ respondents protected themselves while contacting freshwater. The overall positive rate of schistosome nucleic acid among fishermen and boatmen in Yueyang County was $13.81 \%$ (83/601). Multivariate logistic regression analysis showed that age, years of doing current job, times receiving treatment and whether treated in last two years were the main influencing factors of $S$. japonicum infection among this population.

Conclusions: Fishermen and boatmen are still at high risk of infection in China, and gaps existed between knowledge, attitude and practices toward schistosomiasis in this population group. Chemotherapy, health education encouraging behavior change in combination with other integrated approaches to decrease the transmission risk in environments should be strengthened.

\section{Introduction}

Schistosomiasis has been considered to be one of neglected tropical diseases (NTDs) of public health importance in tropical and subtropical areas of the world [1-3]. It is endemic in 78 countries and territories globally with 250 million people infected [4]. People's Republic of China (P.R. China) used to have the highest disease burden of schistosomiasis japonica caused by infection with Schistosoma japonicum. According to the national survey conducted in 1950s, there were 11.60 million cases 
estimated and 1 million infected cattle. Schistosomiasis japonica is acquired when people or domestic animals contact freshwater contaminated with free-swimming cercariae of schistosomes.

During the past sixty years, great efforts have been made to control schistosomiasis through consecutive and vertical control programs by Chinese government. This is especially obvious in the latest decade when schistosomiasis ranks higher priority among four infectious diseases in alliance with HIV/AIDS, tuberculosis and hepatitis $B$ [5], and integrated control strategy was implemented according to the medium and long term national control plan [6-8]. The prevalence and intensity of infection with Schistosoma japonicum in human reduced substantially and the number of endemic provinces has decreased from 12 to $7[9,10]$. Currently, schistosomiasis japonica is mainly endemic in areas surrounding Dongting Lake, Poyang Lake and beaches along Yangtze River [11-14], with fishermen, boatmen and farmers being groups at high-risk of infection and suffering the highest disease burden caused by schistosomiasis [15].

In spite of great achievements obtained for schistosomiasis control in P.R. China, there are still enormous difficulties and challenges during the process towards transmission interruption and elimination of schistosomiasis [16]. Firstly, potential risk factors for resurgence of schistosomiasis japonica still exists and the prevalence of schistosomiasis japonica is easy to rebound in the lake and marshland region due to its unique environment [17]. Secondly, tools used to determine the infection status or prevalence of schistosomiasis in human beings still heavily rely on traditional methods, such as Kato-Katz, which presents an increasingly obvious disadvantage of poor sensitivity, especially in low endemic situations where patients are just slightly infected $[18,19]$. And these false negative and incorrect positive results could eventually contribute to inappropriate decisions made by the government. Thirdly, floating population, especially fishermen and boatmen, is becoming increasingly influential on schistosomiasis transmission according to the national surveillance data [20]. The re-infection with S. japonicum among fishermen and boatmen is still a key issue impeding the transmission interruption or elimination of schistosomiasis in the lake and marshland regions in China [15].

Adequate knowledge, positive attitudes and correct preventive practices of population towards schistosomiasis prevention and control in endemic regions can provide an effectively supportive environment for the success and sustainability of schistosomiasis control [21-23]. Although health education is an important component for schistosomiasis intervention, information on the current infection status and KAPs on schistosomiasis among fishermen and boatmen still remains unclear. Therefore, we conducted a study aimed to assess the KAPs towards schistosomiasis among fishermen and boatmen in particular areas. Infectious status and its influential factors were also identified in this population.

\section{Methods}

Study design 
This study was a cross-sectional survey that assessed knowledge, attitude and practices (KAPs) on schistosomiasis, evaluated the prevalence of schistosomiasis and its risk factors among fishermen and boatmen in study area.

\section{Study area and population}

The study was carried out from October to November in 2017 in Yueyang county, Hunan province, which is located on the eastern bank of Dongting Lake in southern China (Fig.1). Two villages, Lujiao and Matang, in this county were sampled in random by the stratified clustered sampling, in which the prevalence and villages are viewed as stratum and clustering, respectively. As a typical lake and marshland endemic area of schistosomiasis, there are numerous fishermen and boatmen who were considered to be the most at-risk group of $S$. japonicum infection. Fishermen and boatmen in this region commonly live or work on ships for at least four months a year, and their economic incomes mainly comes from fishing, goods transportation. The study population was comprised of either the professional fishermen and boatmen or the sidelines, and all participants involved had not received any antischistosome treatment within a period of six months before the study. The interviews were conducted when their ships berthed in docking spots of Lujiao and Matang in Yueyang County, Hunan Province, P. R. China.

\section{$<$ Figure 1 near here>}

\section{Questionnaire survey}

A pre-tested semi-structured questionnaire was developed after performing a thorough literature review of comparable studies [21, 24-27], to assess the knowledge, attitude and practices (KAP) of participants towards schistosomiasis prevention. This survey was conducted by well-trained interviewers, and was monitored by quality-control supervisors who were arranged for each group before the interviews. The questionnaire consists of four main sections. The first part covered socio-demographic information such as age, gender, education, family income, water-contacting frequency, history of receiving examination and treatment. The second chapter examined participants' knowledge on schistosomiasis, including transmission season, susceptible population, definitive host, transmission mode, intermediate host, clinical manifestations, impacts on children and female, preventive methods and anti-schistosome drug. The third component assessed participants' attitudes towards schistosomiasis prevention. The last section interviewed participants' practices on schistosomiasis prevention. The full questionnaire can be found in online supplementary table S1. 
At least $30 \mathrm{~g}$ stool sample from each participant was collected using a specific container, coded by unique identifiers and transferred to a laboratory in local schistosomiasis control institute. Each sample was scraped out $2 \mathrm{~g}$ with nylon silk and plastic rods to remove the residue from the feces, and then was placed in the cryopreservation tube and kept in the environment of $-20^{\circ} \mathrm{C}$. After all the samples had been pretreated, DNA of Schistosoma japonicum eggs were extracted from feces specimens according to the steps of the DNA extraction kit (QIAGEN, Germany).

Genomic DNA extracted from fecal samples were subjected to 18S-FW (5'-TTCCG ATAAC GAACG AGAC$\left.3^{\prime}\right)$ and 18S-RV (5'-AGCGA TAAAG CCACT ACAAC-3') specific primers reactions amplifying the gene region of $469 \mathrm{bp}$ of $S$. japonicum 18S-rRNA gene fragment [28]. PCR was carried out in a final volume of $50.0 \mu \mathrm{l}$, containing: $2.0 \mu \mathrm{l}$ of template DNA, 25.0 $\mu$ l of dNTPs Mix (TIANGEN BIOTECH Co., Ltd, BEIJING), 1.0 $\mu$ l of upstream and downstream primers respectively, $21.0 \mu \mathrm{l}$ of sterile double distilled water. The PCR conditions were as follows: $5 \mathrm{~min}$ at $94^{\circ} \mathrm{C}$ (initial denaturation), 30 cycles of $20 \mathrm{~s}$ at $94{ }^{\circ} \mathrm{C}, 30 \mathrm{~s}$ at $56^{\circ} \mathrm{C}$, $40 \mathrm{~s}$ at $72^{\circ} \mathrm{C}, 30 \mathrm{cycles}$, and finally $5 \mathrm{~min}$ at $72^{\circ} \mathrm{C}$ (final extension). Meanwhile, negative control (sterile double distilled water) and positive control (worm body tissues DNA) were set up in each batch of experiments. The PCR products were separated on $1.50 \%$ agarose gel. All the positive PCR amplification products encoding 18sRNA were sequenced, and Blast comparison analysis was applied to check the consistency with 18sRNA.

\section{Data management and statistical analysis}

Data collected was entered into a database using EpiData Version 3.0. Data analysis was done using SAS Version 9.4 (Statistical Analysis System, RTI, Cary, North Carolina, USA). The analysis consisted of three parts. Firstly, descriptive statistics were used for demographic characteristics of respondents. Secondly, schistosomiasis control knowledge rates, attitude correct rates, and correct behavior rates were calculated in order to inform the KAPs of S. japonicum among study participants. Finally, Schistosoma nucleic acid positive rate was calculated according to results of PCR amplification and electrophoresis. Risk factors related to the infection of schistosomiasis were analyzed by univariable logistic analysis followed by stepwise regression and multivariate logistic regression model. $P$-values less than 0.05 were considered statistically significant.

\section{Results}

\section{Socio-demographic characteristics}

A total of 753 fishermen and boatmen were enrolled in this cross-sectional study. However, 122 people provided none or insufficient amounts of stool to prepare for the detection by PCR technique, and 30 individuals had no or incomplete questionnaire data. Final study population therefore consisted of 601 
individuals who completed the questionnaire and provided qualified stool samples. The sociodemographic characteristics of the respondents are presented in Table1. The number of males was 345 $(57.40 \%)$ and females was $256(42.60 \%)$. The mean age of respondents was 50.04 years old $( \pm 10.97)$. $59.90 \%$ of respondents (360/601) were sideline fishermen and boatmen while $38.27 \%$ (230/601) were professional boatmen. The majority $(381 / 601,63.39 \%)$ have already done current jobs for 10 to 29 years, followed by those who have done this job for more than 30 years $(128 / 601,21.30 \%)$. More than $95 \%$ respondents received treatment against schistosomiasis at least once.

\section{$<$ Table 1 near here}

\section{Participants' KAPs on schistosomiasis}

Awareness of schistosomiasis knowledge

Of the 601 respondents, $583(97.00 \%)$ mentioned contacting S. japonicum-infested water to be the cause of schistosomiasis. The majority $(575 / 601,95.67 \%)$ of fishermen and boatmen mentioned snail as the intermediate host of Schistosoma japonicum. And 450 (74.88\%) reported to know that the susceptible season for the infection with S. japonicum was from April to October. In terms of the knowledge of symptoms and effects of schistosomiasis, $86.69 \%$ (521/601) of study respondents mentioned fever and diarrhea to be main symptoms for schistosomiasis. 351 (58.40\%) respondents indicated that schistosomiasis would have severe impacts on females, while 377 mentioned schistosomiasis would limit children's physical growth. With respect to preventive activities and treatment for schistosomiasis, the majority $(578,96.17 \%)$ of interviewed fishermen and boatmen mentioned avoiding contact with $S$. japonicum-infested water as an effectively preventive measure for schistosomiasis. Only $288(47.92 \%)$ of study participants mentioned praziquantel to be anti-schistosome drug (Table 2).

\section{$<$ Table 2 near here>}

Attitude of schistosomiasis prevention and control

The majority of respondents $(573 / 601,95.34 \%)$ mentioned that they were willing to install feces containers on their boats. Of the 28 participants who refused to install feces containers, the majority (16, $57.14 \%$ ) complained that fishing boats were too small to accommodate it. Respecting visiting onshore public toilets, 578 respondents $(96.17 \%)$ reported that they would like to use onshore public toilets. 16 of the 22 respondents who were reluctant to use onshore public toilets indicated that it was quite inconvenient to go ashore to find toilets over such a long distance when they were working in the middle of lake. Majority of study respondents presented willingness to accept examination (592, 98.50\%). If 
diagnosed, $592(98.50 \%)$ of the study participants expressed willingness to take medicine regularly following doctors' prescriptions. $89.85 \%$ of respondents (540/601) believed this disease can be prevented, while $432(71.88 \%)$ understood this disease can be cured (Table 3).

Practices of the study participants towards schistosomiasis

Of the 601 respondents, only $40(6.66 \%)$ installed and used fecal containers in the correct way. In terms of protective behaviors when contacting $S$. japonicum-infested water, only 30 of the study respondents reported always wearing rubber shoes, gloves, protective clothing or ointment. Being too troublesome was the reason mentioned by 477 (83.98\%) of those not always protecting themselves when contacting freshwater in Dongting Lake. Only 196 (32.61\%) of the participants reported to always defecate in onshore public toilets (Table 3 ).

$<$ Table 3 near here $>$

\section{S. japonicum infection status and related risk factors}

Among 601 participants who provided qualified stool samples, 83 were detected as positives by PCR method, with $13.81 \%(83 / 601)$ as the positive rate of schistosome nucleic acid. PCR amplification products of all the positive samples were sequenced, and results showed that the fragment was $469 \mathrm{bp}$, which was consist with the target DNA both in length and sequence $\triangle F i g$ 2Х. Participants who are aged less than 30 years old, doing current jobs for more than 30 years and never received treatment against schistosomiasis presented the highest PCR positive rates than other subgroups. The positive rate of Schistosoma nucleic acid in males $(14.78 \%, 51 / 345)$ was higher than that of females $(12.50 \%, 32 / 256)$, but no statistics difference was detected $\nabla \chi^{2}=0.643 \rrbracket p=0.423 \rrbracket$.

\section{$<$ Figure 2 near here>}

Based on univariate logistic regression analysis, nine independent variables were included in the multivariate logistic regression, which were in turn age, occupation, economic conditions, years of doing current job, infection history, diagnostic method, disease category, times received treatment, whether received anti-schistosome treatment in 2015-2017. The results of multivariate logistic regression analysis showed that age, years of doing current job, times received treatment and whether received antischistosome treatment in 2015-2017 were significantly associated with the infection of S. japonicum (Table 4). 
The results indicated that respondents aged in groups of 40-, 50- and 60-had significantly lower odds of getting positive results than younger groups determined by PCR (OR=0.163, 95\% Cl: 0.041-0.644; $\mathrm{OR}=0.058,95 \% \mathrm{Cl}: 0.014-0.239 ; \mathrm{OR}=0.012,95 \% \mathrm{Cl}$ : 0.002-0.065). Logistic regression analysis also revealed that the fishermen and boatmen who have done current job for 10-29 years and over 30 years are more likely to be PCR positives with OR=4.162 (95\%Cl: 1.461-11.856), OR= 18.684 (95\% Cl: 5.430-64.295), respectively.

In addition, the respondents who received treatment 6-10 times, more than 10 times showed lower odds of presenting positive results determined by PCR (OR=0.156 (95\%Cl: $0.035-0.689), \mathrm{OR}=0.100$ (95\% Cl: 0.021-0.476)), when comparing with those never receiving treatment against schistosomiasis ever before. Also, respondents who received treatment during 2015-2017 were more likely to have negative results determined by PCR (OR=0.479, 95\% Cl: 0.246-0.930).

\section{$<$ Table 4 near here>}

\section{Discussion}

In this study, the KAPs survey was conducted to explore the level of KAPs of fishermen and boatmen on schistosomiasis in Yueyang County of Hunan Province, P. R. China. Infection status of schistosomiasis in this special group was determined by a sensitive method based on nucleic acid detection and risk factors related to infection were identified through logistic regression models. This study aims to provide information for the design and development of integrated control strategy against schistosomiasis in China.

Our findings showed that the overall awareness of schistosomiasis control knowledge among study participants was considered to be relatively satisfactory, which attributes to the persistent health education conducted for many years. More than $90 \%$ respondents knew the cause of getting infection, intermediate host of schistosomes and preventive methods, while majority of the respondents knew this disease, its clinical manifestations and transmission mode. However, less than $70 \%$ respondents know the medication against schistosomiasis as well as its impacts on children and female. We also found that majority of the respondents had positive attitudes towards schistosomiasis prevention. More than $95 \%$ respondents had the willingness to install feces containers on their boats, using onshore public toilets, and taking medicine against schistosomiasis, which would benefit to the control of schistosomiasis in this population. Nearly $90 \%$ participants believed that schistosomiasis was preventable and $71.88 \%$ respondents trusted schistosomiasis could be cured.

Despite sufficient knowledge and good attitudes towards schistosomiasis prevention, behavioral practices of fishermen and boatmen were quite unsatisfactory on the contrary. People preferred to receive examination and treatment, rather than to prevent. Only $6.66 \%$ respondents had installed latrine on their boats, and only $32.61 \%$ respondents defecated in the onshore public toilets. There are many reasons 
given by interviewed fishermen and boatmen. On the one hand, for example, fishing boats are too small to install fecal containers and stools are easy to overflow out of containers because of the instability on board. On the other hand, feces-induced smell makes containers intolerable on board. In addition, only $4.99 \%$ respondents protected themselves when contacting freshwater. These respondents complained it is troublesome and inconvenient to wear protections when fishing or working on the water. Adequate knowledge and positive attitudes didn't convert to behavior changes effectively, mainly due to the strong economic drives of their works, which was corresponding to former studies [21, 29]. The gaps observed between knowledge, attitude and practices indicated that effective methods and enormous efforts should be made to better address the problem of transmission caused by behavior approaches. First of all, appropriate health promotion activities should be provided to this high-risk population. Secondly, existing intervention approaches should be improved or modified to be more accessible and attractive to fishermen and boatmen. Finally, integrated approaches to eliminate the transmission risk of schistosomiasis in freshwater body should be further explored and strengthened [13]. In addition, the prohibition of fishing activities is going to be implemented along the Yangtze River, which can be literally helpful for current situation. Meanwhile, effective monitoring system needs to be established and run efficiently to guarantee this policy.

In P. R. China, a two-step detection pattern with serological screening first followed by stool examination only for serological positives is applied for field diagnosis of schistosmiasis in China [15]. This pattern presented great advantages in the early age because of its enormous capacity of large-scale screening and examination. However, with the persistent implementation of integrated control strategy for schistosomiasis, the prevalence and intensity of schistosomiasis in China have reached a very low level in history $[19,30]$. The infected fishermen and boatmen can be easily misdiagnosed by traditional methods such as Kato-Katz method or miracidia hatching technique, and therefore not targeted for chemotherapy, which brings great potential risks to the elimination of schistosomiasis in China [31, 32]. As a classic nucleic acid amplification technique, PCR method has a varied of advantages over stool examination, such as higher sensitivity and specificity, low cost of reagents and universal equipment in labs, which was considered to hold great potentials of application for schistosomiasis detection in the future [33]. It was reported that only 2.16 eggs per gram feces were needed to detect schistosomiasis by PCR method [33].

This article found a high burden of schistosomiasis in fishermen and boatmen in Yueyang County by PCR method $(83 / 691,13.81 \%)$, which is higher than the average prevalence obtained from national surveillance system[15, 21, 34]. Following explanations as well as considerations are therefore initiated for discussion. Firstly, the study population generally spend most of their lives on board, they therefore contact with S. japonicum-infested water frequently, which is thought to be the main reason for their vulnerability to infection and re-infection of schistosome [32, 35]. Secondly, interventions against schistosomiasis were not accessible to fishermen and boatmen due to their high mobility [24, 36]. Among the 601 respondents in our research, 25 (4.16\%) of 601 never received treatment and $220(36.61 \%)$ only received treatment two years ago before our study. Thirdly, our study used nucleic acid-based detection method rather than traditional methods. The PCR technique used in this article performed excellent 
sensitivity as it could detect $10 \mathrm{pg} / \mu \mathrm{l}$ schistosome genome DNA based on internal assessment, and great specificity reflected by DNA sequencing of PCR products. PCR technique, therefore, can benefit the diagnosis and treatment on individuals with low-level infections and thus promote the elimination of schistosomiasis in P. R. China.

The results from logistic regression showed that four variables including age, years of doing current job, times received treatment in history and whether received treatment in recent two years were significantly associated with PCR results which were used to measure the infection of S. japonicum. Younger fishermen and boatmen are more likely to be infected by schistosomes, which have the similar results in previous research [24]. As main labor forces of their families, young fishermen and boatmen are more likely to be involved in fishing or household chores, which signifies higher frequency and greater level of exposure to $\mathrm{S}$. japonicum-infested water, and therefore have more opportunities to get infected. In addition, there are also studies showing that aged fishermen and boatmen with long-term exposure to water can produce a kind of acquired immunity towards Schistosoma in their bodies, which protects them to a certain degree [5]. We also found that years of doing current jobs were also strongly associated with the infection of S. japonicum. Specifically, the more years doing current job, the higher risk of having positive results determined by PCR. Moreover, our findings showed that the prevalence decreased significantly with treatment times extended among this population. The respondents who have never been treated ever before and those who only been treated once to five times presented positive rates of $32.00 \%$ and $17.13 \%$, respectively. Also, prevalence was higher in the subgroup which have not received any treatment in recent two years. These results all indicated that chemotherapy is still an effective way to control schistosomiasis in this population, who keeps being at high risk of infection in lake and marshland regions and serves as the main obstacle of schistosomiasis elimination in P.R. China [25].

One of the limitations in our study is that not all risk factors were considered as its entirety in the analysis, e.g. some of economic and historical elements, which may lost some of information in the study. Secondly, the 152 fishermen and boatmen who were excluded out of the study might have an impact on the study results to some extent. Furthermore, molecular diagnosis technology itself is continuously developing, and PCR technology is gradually improved. Therefore, it is promising for the application of PCR to the epidemiological study in neglected tropical diseases, and to understand the prevalence of disease accurately.

\section{Conclusions}

Our findings show that fishermen and boatmen remain to be the most vulnerable population to get infection of schistosomes in endemic areas, who serve as an enormous obstacle of hindering the progress of schistosomiasis elimination in P. R. China. Among this population, younger fishermen and boatmen with longer occupational history as well as less treatments, particularly in recent two years, are at increased risk of getting infection. In addition, gaps literally exist in this population between knowledge, attitude and practices towards schistosomiasis prevention. We therefore suggest that 
enhanced chemotherapy, health promotion activities encouraging behavior change and other integrated approaches should be combined and strengthened to reduce the scale of this high-risk group.

\section{Abbreviations}

P. R. China: People's Republic of China; KAPs: knowledge, attitude and practices; PCR: Polymerase Chain Reaction; NTDs: neglected tropical diseases; OR: odd ratio; $95 \% \mathrm{Cl}$ : $95 \%$ confidence interval.

\section{Declarations}

\section{Ethics approval and consent to participate}

Ethical clearance had been granted by the Ethics Committee of the National Institute of Parasitic Diseases, Chinese Center for Disease Control and Prevention in Shanghai, P.R. China (20170717). During these meetings, the objectives of the study, the study procedures, sampling, study benefits, and potential risks and discomforts were orally explained and informed to all participants. All adult participants gave informed consent, and informed consent for all children who participated in the study was sought from parents or legal guardians. Assent was sought from all participants who were also informed of their rights to refuse to participate in this study and to withdraw from the study at any time. Participants with positive result of PCR received free treatments with a standard dose of praziquantel.

\section{Consent for publication}

Not applicable.

\section{Availability of data and materials}

All relevant data are contained within the paper and its additional files.

\section{Competing interests}

The authors declare that they have no competing interests.

\section{Funding}

This study was financially supported by the National Special Science and Technology Project for Major Infectious Diseases of China (No. 2018ZX10101002-002, 2012ZX10004-220) and The National Key Research and Development Program of China (2016YFC1202000). 


\section{Authors' Contributions}

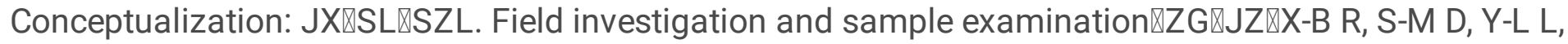
Z-Q Q. Supervision: JX, S-Z L, SL, X-N Z. Writing-original draft: ZG, S-M D, JX. Writing-review \& editing: JX. All authors read and approved the final manuscript.

Conceived and designed experiments: JX. Performed the experiments: ZG SMD. Analyzed the data: ZG. Wrote the paper: ZG SMD.

\section{Acknowledgments}

The authors would like to acknowledge the technicians of schistosomiasis control station of Yueyang County, who provided invaluable assistance with data collection and stool samples' collection. We are grateful to the schistosomiasis control institute of Hunan province, for permission and support.

\section{Authors' information}

\section{Affiliations}

National Institute of Parasitic Diseases, Chinese Center for Disease Control and Prevention; Key Laboratory of Parasite and Vector Biology, National Health Commission; WHO Collaborating Centre for Tropical Diseases; National Center for International Research on Tropical Diseases, Shanghai, People's Republic of China

Zhou Guan, Si-Min Dai, Zhi-Qiang Qin, Yin-Long Li, Shan Lv, Shi-Zhu Li, Xiao-Nong Zhou, Jing Xu* Hunan Institute of Schistosomiasis Control, Yueyang, People's Republic of China Jie Zhou

Yueyang County Office for Preventive and Control on Schistosomiasis, Yueyang, People's Republic of China

Xiao-Bing Ren

\section{References}

1. Bruun B, Aagaard-Hansen J. The Social Context of Schistosomiasis and Its Control: An Introduction and Annotated Bibliography. World Health Organization on be-half of the Special Programme for 
Research and Training in Tropical Diseases. 2008.

2. Steinmann, Peter, Keiser, Jennifer, Robert, Tanner, et al. Schistosomiasis and water resources development: systematic review, meta-analysis, and estimates of people at risk. Lancet Infectious Diseases. 2006;6(7):411-25.

3. Crompton DW. How much human helminthiasis is there in the world? Journal of Parasitology. 1999;85(3):397-403.

4. World Health Organization.. Schistosomiasis: progress report 2001-2011 and strategic plan 20122020. 2013. Available from:

http://www.who.int/iris/bitstream/10665/78074/1/9789241503174_eng.pdf. Accessed 6 August 2018.

5. Xu JF, Xu J, Li SZ, Jia TW, Huang XB, Zhang HM, et al. Transmission Risks of Schistosomiasis Japonica: Extraction from Back-propagation Artificial Neural Network and Logistic Regression Model. PLoS Neglected Tropical Diseases,7,3(2013-3-21). 2013;7(3):e2123.

6. Wang LD, Chen HG, Guo JG, Zeng XJ, Hong XL, Xiong JJ, et al. A strategy to control transmission of Schistosoma japonicum in China. New England Journal of Medicine. 2009;360(2):121.

7. He JC, Wang TP, Zhang SQ, Gao FH, Zhang GH, Yang WP, et al. Evaluation of mid-term effectiveness of medium-and-long-term programme for prevention and control of schistosomiasis in Anhui Province. Chinese Journal of Schistosomiasis Control. 2011;23(3):249.

8. Zeng XJ, Chen HG, Hong XL, Hu ZH, Jiang WS, Hu SZ, et al. Evaluation on medium-term effect of schistosomiasis comprehensive control strategy based on infectious source control in Poyang Lake area. Chinese Journal of Schistosomiasis Control. 2012;24(4):382-6.

9. Zhou XN, Guo JG, Wu XH, Jiang QW, Zheng J, Dang H, et al. Epidemiology of schistosomiasis in the People's Republic of China, 2004. Emerging Infectious Diseases. 2007;13(10):1470.

10. Zhou XN, Wang LY, Chen MG, Wu XH, Jiang QW, Chen XY, et al. The public health significance and control of schistosomiasis in China--then and now. Acta Tropica. 2005;96(2-3):97.

11. Sun LP, Wang W, Zuo YP, Hong QB, Du GL, Ma YC, et al. A multidisciplinary, integrated approach for the elimination of schistosomiasis: a longitudinal study in a historically hyper-endemic region in the lower reaches of the Yangtze River, China from 2005 to 2014. Infectious diseases of poverty. 2017;6(1):56.

12. Colley DG, Bustinduy AL, Secor WE, King CH. Human schistosomiasis. Lancet.383(9936):2253-64.

13. Chitsulo L, Loverde P, Engels D. Focus: Schistosomiasis.2(1):12-3.

14. Abou-Zeid AHA, Abkar TA, Mohamed RO. Schistosomiasis and soil-transmitted helminths among an adult population in a war affected area, Southern Kordofan state, Sudan.5(1).

15. Guan Z, Lu S, Li SZ, Dang H, Zhang LJ, Xu J. Analysis on the situation of schistosome infections in floating population in national schistosomiasis surveillance sites of China. Chinese journal of schistosomiasis control. 2018;30(2):124-30. 
16. Zhou XN, Bergquist R, Leonardo L, Yang GJ, Yang K, Sudomo M, et al. Schistosomiasis japonica: control and research needs. Advances in Parasitology. 2009;72:145.

17. Zhao GM, Zhao Q, Jiang QW, Chen XY, Wang LY, Yuan HC. Surveillance for schistosomiasis japonica in China from 2000 to 2003. Acta Tropica. 2005;96(2-3):288-95.

18. Guan Z, Lu S, Li SZ, Xu J. Endemic status of schistosomiasis in floating population and control challenges in P.R. China. Chinese journal of schistosomiasis control. 2017;35(6):598-603.

19. Xu J, Guan ZX, Zhao B, Wang YY, Cao Y, Zhang HQ, et al. DNA detection of Schistosoma japonicum: diagnostic validity of a LAMP assay for low-intensity infection and effects of chemotherapy in humans. PLoS neglected tropical diseases. 2015;9(4):e0003668.

20. Huang SY, Lin RX, Zhang QM, Deng ZH, Zhang XC, Huo LC. Analysis of schistosomiasis surveillance in floating population in Guangdong,2005-2009. South China Journal of Preventive Medicine. 2010.

21. Odiere MR, Rawago FO, Ombok... M. High prevalence of schistosomiasis in Mbita and its adjacent islands of Lake Victoria, western Kenya.5(1):278.

22. Norton AJ, Gower CM, Lamberton PHL, Webster BL, Lwambo NJS. Genetic Consequences of Mass Human Chemotherapy for Schistosoma mansoni: Population Structure Pre- and Post-Praziquantel Treatment in Tanzania.

23. Hotez PJ, Fenwick A. Schistosomiasis in Africa: An Emerging Tragedy in Our New Global Health Decade. PLoS neglected tropical diseases.3(9):e485.

24. Yu XL, Zhou J, He YK, Huang MZ, Li YS. Influence factors of Schistosoma japonicum infection among fishermen in eastern Dongting Lake Region. Chinese Journal of Parasitology \& Parasitic Diseases. 2013;31(4):307-9.

25. Zhang J, Li ZJ, Qiu L, Li D, Chen JJ, Xie H, et al. Development and application of communication materials for participatory health education of schistosomiasis in fishermen and boatmen of Poyang Lake region. Chinese journal of schistosomiasis control. 2016;28(1):58-61.

26. Zhou J, Huang CY, He YK, Du YQ, Yu XL, Wang YY, et al. Epidemiological evaluation of schistosomiasis in migrant fishermen in Dongting Lake region. Chinese Journal of Schistosomiasis Control. 2010.

27. Cao CL, Bao ZP, Zhu HQ, Yu Q, Li SZ, Wang Q, et al. Analysis of schistosomiasis control requirements in boat fishermen in lake regions. Chinese Journal of Schistosomiasis Control. 2010.

28. Chen JH, Wen LY, Zhang XZ, Zhang JF, Yu LL, Hong LD. Development of a PCR assay for detecting Schistosoma japonicum-infected Oncomelania hupensis. Chinese Journal of Parasitology \& Parasitic Diseases. 2006;24(3):204.

29. Cawston FG. Schistosomiasis in Southern Africa. 2010;127(6):509-13.

30. Zhou XN, Xu J, Chen HG, Wang TP, Huang XB, Lin DD, et al. Tools to support policy decisions related to treatment strategies and surveillance of Schistosomiasis japonica towards elimination. PLoS neglected tropical diseases. 2011;5(12):e1408. 
31. Zhang LJ, Xu ZM, Qian YJ, Dang H, Lu S, Xu J, et al. Endemic status of schistosomiasis in People's Republic of China in 2015. Chinese Journal of Schistosomiasis Control. 2016;29(3):273.

32. Yi DH, Yi P, Liu ZC, Li YS, Quan MZ, Xiao SY. Practice and thought of schistosomiasis control with an emphasis on control sources of infection in Dongting Lake area. Chinese Journal of Schistosomiasis Control. 2009.

33. Pontes LA, Diasneto E, Rabello A. Detection by polymerase chain reaction of Schistosoma mansoni DNA in human serum and feces. American Journal of Tropical Medicine \& Hygiene. 2002;66(2):15762.

34. Malenganisho WLM, Magnussen P, Friis H, Siza J, Vennervald BJ. Schistosoma mansoni morbidity among adults in two villages along Lake Victoria shores in Mwanza District, Tanzania. Transactions of the Royal Society of Tropical Medicine \& Hygiene. 2008;102(6):532-41.

35. Ni L, Li PY, Mao XZ. Four predicaments facing Dongting Lake fishermen got ashore. Journal of Hunan Agricultural University. 2010.

36. Fabri RL, Florêncio JR, Pinto NDCC, Mattos ACA, Coelho PMZ, Vasconcelos EG, et al. Chromatographic Fingerprint Analysis and Effects of the Medicinal Plant Species Mitracarpus frigidus on Adult Schistosoma mansoni Worms. Biomed Research International. 2014;2014(8):941318.

\section{Tables}

Table 1 Major socio-demographic characteristics of respondents attending KAPs survey and provided stool samples

\begin{tabular}{llll}
\hline Variables & Category & Frequency & Percentage (\%) \\
\hline Gender & male & 345 & 57.40 \\
& female & 256 & 42.60 \\
\cline { 2 - 4 } Occupation & Professional fisherman & 11 & 1.83 \\
\cline { 2 - 4 } & Professional boatman & 230 & 38.27 \\
\cline { 2 - 4 } & sideline fisherman and boatman & 360 & 59.90 \\
\hline \multirow{2}{*}{ Age group } & $<30$ & 21 & 3.49 \\
\cline { 2 - 4 } (years) & $30 \sim$ & 54 & 8.99 \\
& $40 \sim$ & 228 & 37.94 \\
& $50 \sim$ & 184 & 30.62 \\
\hline \multirow{2}{*}{ Education } & Below primary school & 114 & 18.97 \\
& Primary school & 57 & 9.48 \\
\cline { 2 - 4 } & Junior middle school & 324 & 27.79 \\
\cline { 2 - 4 } & High school and higher education & 53 & 83.91 \\
\hline
\end{tabular}

Table 2 Knowledge of schistosomiasis among respondents 


\begin{tabular}{lccc}
\hline Knowledge regarding schistosomiasis & Response & Frequency & Percentage \\
& & & $(\%)$ \\
\hline Transmission season & Right & 450 & 74.88 \\
Susceptible population & Wrong & 151 & 25.12 \\
\cline { 2 - 3 } & Right & 433 & 72.05 \\
\hline Final host & Wrong & 168 & 27.95 \\
\hline Cause of infection & Right & 451 & 75.04 \\
\hline Intermediate host & Wrong & 150 & 24.96 \\
\hline Main symptoms & Right & 583 & 97.00 \\
\hline Effects on children & Wrong & 18 & 3.00 \\
\hline Effects on women & Right & 575 & 95.67 \\
\hline Medicine used for treatment & Wrong & 26 & 4.33 \\
\hline Preventive measures & Right & 521 & 86.69 \\
\hline & Wrong & 80 & 13.31 \\
\hline & Right & 377 & 62.73 \\
\hline & Wrong & 224 & 37.27 \\
\hline & Right & 351 & 58.40 \\
\hline & Wrong & 250 & 41.60 \\
\hline & Right & 288 & 47.92 \\
\hline & Wrong & 313 & 52.08 \\
\hline & Right & 578 & 96.17 \\
\hline & Wrong & 23 & 3.83 \\
\hline
\end{tabular}

Table 3 Attitude and practices toward schistosomiasis prevention and control among the study participants

\begin{tabular}{llll}
\hline Attitude and practices toward schistosomiasis & Category & Frequency & Percentage \\
& & & $(\%)$ \\
\hline Willingness to install feces containers & Yes & 573 & 95.34 \\
Willingness to use onshore public toilets & No & 28 & 4.66 \\
\cline { 2 - 3 } & Yes & 578 & 96.17 \\
\hline Willingness to accept examination & No & 23 & 3.83 \\
\hline Willingness to take medicine & Yes & 592 & 98.50 \\
\cline { 2 - 4 } & No & 9 & 1.50 \\
\hline Belief that schistosomiasis could be prevented & Yes & 592 & 98.50 \\
\cline { 2 - 4 } & No & 9 & 1.50 \\
\hline Belief that schistosomiasis could be cured & Yes & 540 & 89.85 \\
\cline { 2 - 4 } Install and use fecal containers & No & 61 & 10.15 \\
\hline Defecate in public toilets onshore & Yes & 432 & 71.88 \\
& Yes & 169 & 28.12 \\
\hline Protection when contacted water & No & 561 & 6.66 \\
\hline & Yes & 196 & 32.34 \\
\hline
\end{tabular}


Table 4 Multiple logistic regression analysis of variables associated with $S$. japonica among study participants

\begin{tabular}{lll}
\hline Risk factors & Positive rate determined by PCR & OR $(95 \% \mathrm{Cl})$ \\
\multicolumn{3}{c}{$($ No. positives/No. examined) } \\
\hline $\begin{array}{l}\text { Age group (years) } \\
<30\end{array}$ & $38.10 \%(8 / 21)$ & 1 \\
$30-$ & $27.78 \%(15 / 54)$ & $0.318(0.075-1.354), P=0.1212$ \\
$40-$ & $15.35 \%(35 / 228)$ & $0.163(0.041-0.644), P=0.0096^{*}$ \\
$50-$ & $10.33 \%(19 / 203)$ & $0.058(0.014-0.239), P<.0001^{*}$ \\
$60-$ & $5.26 \%(6 / 114)$ & $0.012(0.002-0.065), P<.0001^{*}$ \\
Years of doing current job & \\
$<10$ & $7.61 \%(7 / 92)$ & 1 \\
$10-$ & $12.86 \%(49 / 381)$ & $4.162(1.461-11.856), P=0.0076^{*}$ \\
$30-$ & $21.09 \%(27 / 128)$ & $18.684(5.430-64.295), P<.0001^{*}$ \\
Treatment times & & 1 \\
0 & $32.00 \%(8 / 25)$ & $0.265(0.064-1.095), P=0.0666$ \\
$1-$ & $17.13 \%(37 / 216)$ & $0.156(0.035-0.689), P=0.0142^{*}$ \\
$6-$ & $12.26 \%(26 / 212)$ & $0.100(0.021-0.476), P=0.0038^{*}$ \\
$10-$ & $8.11 \%(12 / 148)$ & \\
Treatment in 2005-2017 & & 1 \\
No & $18.18 \%(40 / 220)$ & $0.479(0.246-0.930), P=0.0298^{*}$ \\
\hline Yes & $11.29 \%(43 / 381)$ & \\
\hline
\end{tabular}

OR, odds ratio. $\mathrm{Cl}$, Confidence interval. *Significant association $(\mathrm{P}<0.05)$

Figures 


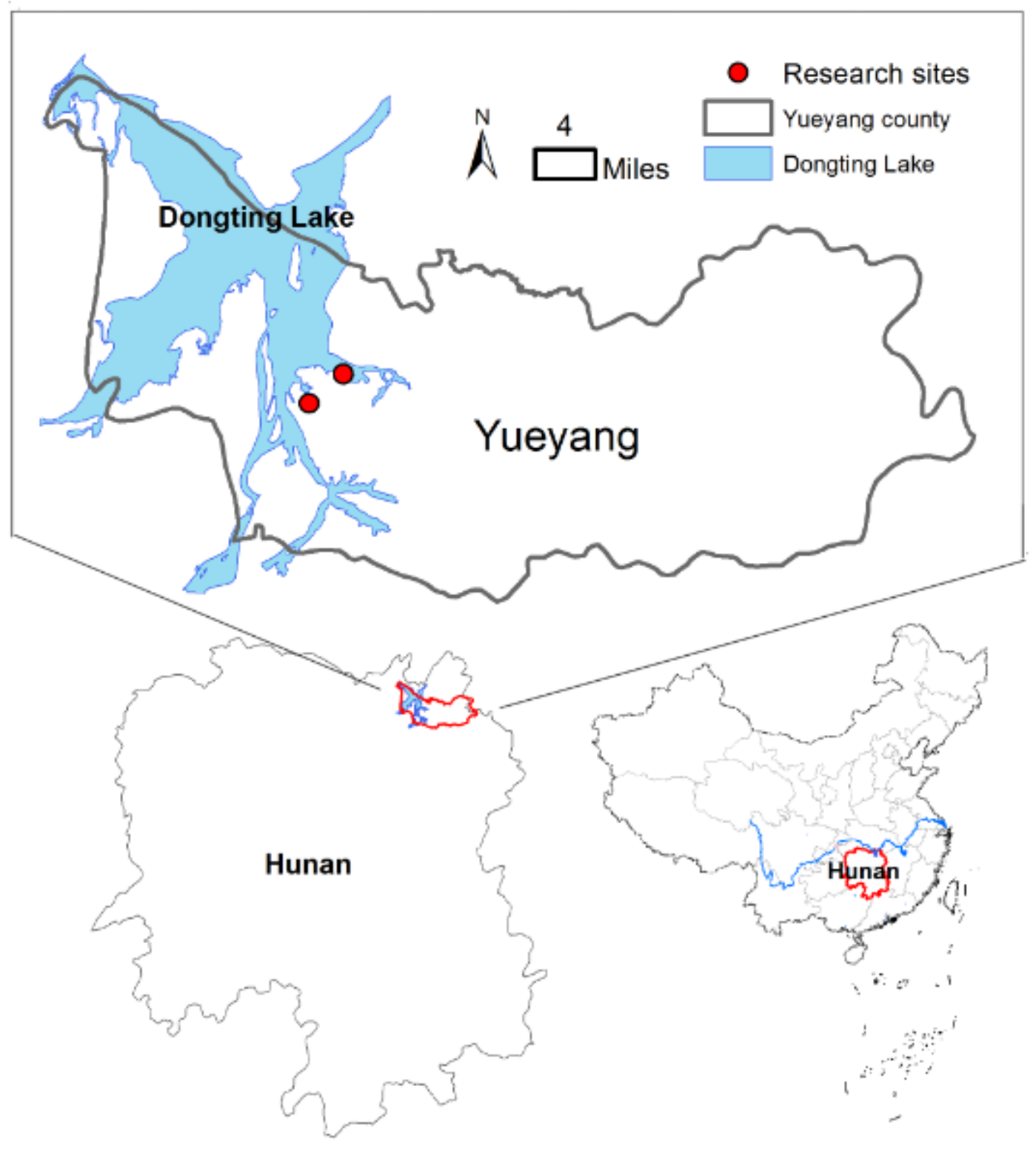

\section{Figure 1}

Location of Study Area in Yueyang County, Hunan Province. Note: The designations employed and the presentation of the material on this map do not imply the expression of any opinion whatsoever on the part of Research Square concerning the legal status of any country, territory, city or area or of its authorities, or concerning the delimitation of its frontiers or boundaries. This map has been provided by the authors. 


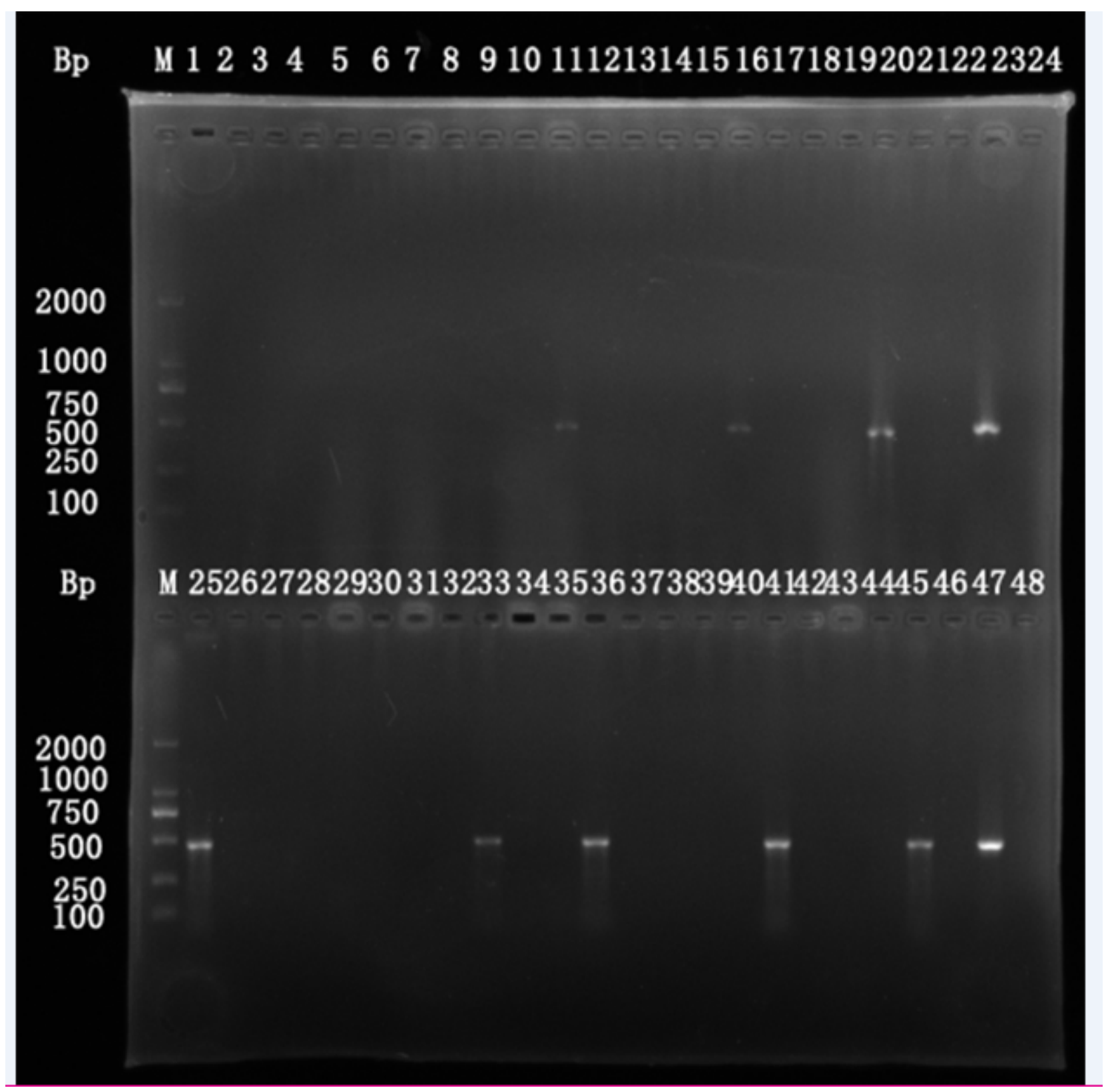

Figure 2

Results of PCR detection of the respondents

\section{Supplementary Files}

This is a list of supplementary files associated with this preprint. Click to download.

- renamed8be02.jpg 